\title{
Vom Erlebnis zum Ergebnis: Zur Wirkungsweise abenteuer- und erlebnispädagogischer Jugendfreizeiten
}

\author{
Michael Mutz $\mathbb{D} \cdot$ Johannes Müller
}

Eingegangen: 11. Februar 2019 / Überarbeitet: 21. April 2020 / Angenommen: 16. Juli 2020 / Online publiziert: 6. August 2020

(C) Der/die Autor(en) 2020

Zusammenfassung Die Forschung über abenteuer- und erlebnispädagogische Programme hat vielfach auf positive Effekte z. B. für Selbstwirksamkeit, Kompetenzerwerb oder psychosoziale Gesundheit hingewiesen. Weniger gut erforscht ist hingegen die Frage, unter welchen Bedingungen diese Effekte zu Stande kommen bzw. welche Erfahrungen mit welchen Veränderungen assoziiert sind. Anknüpfend an erlebnispädagogische Theorien gehen wir davon aus, dass a) die Neuartigkeit der Erfahrungen, b) die erfolgreiche Selbstüberwindung, c) das Kompetenzerleben und d) die Qualität der Einbindung in die Gruppe zentrale Erfolgsbedingungen darstellen. Empirisch prüfen wir die Bedeutung dieser Bedingungen in einem Prätest-PosttestDesign bei Jugendlichen ( $N=76 ; 63 \%$ männlich, $37 \%$ weiblich) im Alter von 13 bis 20 Jahren, die an einer 10-tägigen erlebnispädagogischen Ferienfreizeit teilnahmen. Die Ergebnisse zeigen zunächst, dass sich im Durchschnitt positive Veränderungen in der Selbstwirksamkeitserwartung, im Selbstwertgefühl und im emotionalen Wohlbefinden im Verlauf des Programms ergeben haben. Diese Effekte sind besonders bei den Jugendlichen zu beobachten, die sich bei den Programmaktivitäten als sehr kompetent erlebten. Punktuell hängen positive Veränderungen auch mit der Neuartigkeit der Aktivität und der Qualität des Gemeinschaftserlebens zusammen. Der oft in der Theoriedebatte fokussierte Aspekt der Selbstüberwindung scheint weniger relevant zu sein.

Schlüsselwörter Abenteuerpädagogik · Erfahrungslernen · Selbstwirksamkeit · Selbstwertgefühl

Prof. Dr. M. Mutz ( $\varangle) \cdot$ Dr. J. Müller

Institut für Sportwissenschaft, Justus-Liebig-Universität Gießen, Kugelberg 62, 35394 Gießen, Deutschland

E-Mail: michael.mutz@sport.uni-giessen.de

Dr. J. Müller

E-Mail: johannes.mueller@sport.uni-giessen.de 


\title{
From experiences to outcomes: Conditions and mechanisms of effective outdoor and adventure education camps for youths
}

\begin{abstract}
Research on outdoor and adventure education programs provided ample evidence that these programs have positive effects on e.g. self-efficacy, competency acquisition or psychosocial health. However, far less explored is the question under which conditions these effects are achieved. Hence, this paper focuses on the mechanisms of change. Following adventure education theories, we assume that a) the novelty of experiences, b) the successful self-conquest, c) the experience of mastery and d) the quality of involvement into the group are crucial conditions for success. We empirically test the significance of these conditions within a pretest-posttest design using questionnaire data from youths $(N=76 ; 63 \%$ male, $37 \%$ female $)$ in the age range of 13 to 20 years, who took part in a 10-day outdoor and adventure education camp. With regard to self-efficacy, self-esteem, and emotional well-being, our findings show positive changes, on average, in the course of the program. Larger effects occur among those who had mastery experiences during the program. Positive changes also occasionally correlate with the novelty of activities and the sense of belonging in the group. The aspect of self-conquest often focused in theoretical debates seems to be less relevant.
\end{abstract}

Keywords Adventure education $\cdot$ Experiential learning $\cdot$ Self-efficacy $\cdot$ Self-worth

\section{Einführung}

Abenteuer- und erlebnispädagogische Programme erfahren seit Jahrzehnten eine große und bis heute ungebrochene Popularität. Seit den Anfängen der „Outward Bound"-Bewegung in den 1940er Jahren, als deren Wegbereiter Kurt Hahn gilt, nimmt die Erlebnispädagogik inzwischen in Bildungseinrichtungen, der außerschulischen Sozial- und Jugendarbeit, aber auch im Freizeitbereich einen festen Platz ein. Neben „klassischen“ Zielen, wie der Persönlichkeitsentwicklung, dem Erwerb sozialer Kompetenzen und der Förderung des Gruppenzusammenhalts (Heckmair und Michl 2008), werden inzwischen vor dem Hintergrund einer verstärkten Technisierung des Alltags und einer damit verbundenen Distanz zur Natur auch Naturerleben und Verständnis für Umweltschutz als Bildungsziele dieser Projekte verstärkt diskutiert (Öhman und Sandell 2016). Die Zielgruppen erlebnispädagogischer Maßnahmen sind dabei vielfältig und nicht ausschließlich auf Heranwachsende beschränkt. In der Literatur finden sich Beiträge über erlebnispädagogische Schulfahrten (Reuker 2009; Mutz und Müller 2016), gezielte Interventionen für Delinquente (Brand 2002; Gillis und Gass 2010), sozial Benachteiligte (Amesberger 1998) oder Menschen mit körperlichen oder psychischen Beeinträchtigungen (Schell et al. 2012; Kinne und Theunissen 2013).

Die Wirksamkeit erlebnispädagogischer Initiativen ist auf nationaler und internationaler Ebene in der Vergangenheit vielfach wissenschaftlich untersucht worden. Überblicksartikel und Meta-Analysen weisen übereinstimmend auf die Wirksamkeit hin, wobei die Stärke der Effekte je nach Zielsetzungen, Programminhalten oder 
Merkmalen der Teilnehmenden erheblich variieren (Hattie et al. 1997; Ewert und McAvoy 2000; Gillis und Speelman 2008; Stott et al. 2015). Einzelstudien jüngeren Datums belegen insbesondere positive Veränderungen im Hinblick auf Selbstwirksamkeit (Kümmel et al. 2008; Gillespie und Allen-Craig 2009; Gehris et al. 2010; Schell et al. 2012), Selbstvertrauen (Gatzemann et al. 2008: Hunter et al. 2013), Resilienz (Hayhurst et al. 2015; Scarf et al. 2017), Befindlichkeit und psychische Gesundheit (Boeger et al. 2006; Mutz und Müller 2016; Mutz et al. 2019), prosoziales Verhalten (Reuker 2009) sowie Gruppenzusammenhalt (Moch 2002; Sommer 2005; Goldenberg et al. 2005; Reichmann 2011).

Im Gegensatz zu der umfangreichen und ausdifferenzierten Studienlage zur Wirksamkeit erlebnispädagogischer Programme gibt es nur wenige empirische Beiträge, die sich genauer mit der Wirkungsweise dieser Maßnahmen auseinandersetzen. Wenngleich Priest (1999) bereits vor zwanzig Jahren forderte, künftig vor allem die Bedeutung spezieller Programmelemente erlebnispädagogischer Interventionen zu untersuchen, d.h. vor allem die Zusammenhänge zwischen der Programmwirksamkeit und z.B. der Dauer, des Inhalts, des Orts sowie des Settings der jeweiligen Programme zu erforschen, hat sich bis dato wenig getan: So resümieren z. B. Heekerens (2018) und Fengler (2017), dass in der erlebnispädagogischen Forschung noch immer mehr über die Wirksamkeit als über die Wirkungsmechanismen der Programme bekannt ist und in der Erlebnispädagogik die Ergebnisforschung gegenüber der Prozessforschung dominiert. Dies wird damit begründet, dass die Prozessforschung weitaus schwieriger und anspruchsvoller als die reine Ergebnisforschung ist. Vor diesem Hintergrund fordert Fengler (2017) eine stärkere Beforschung theoretisch hergeleiteter Wirkprinzipien bzw. Heekerens (2006, S. 49) eine ,verschränkte Prozess-Ergebnis-Forschung“". Priest (1999) plädiert ebenfalls dafür, die Programminhalte und -merkmale mit Veränderungen bei Teilnehmenden in Beziehung zu setzen. Gleichwohl thematisiert er die Schwierigkeiten dieser Forschung, wie z. B. sehr kleine Gruppengrößen bei erlebnispädagogischen Projekten; die Schwierigkeit, randomisierte Kontrollgruppen zu etablieren oder die Unmöglichkeit, die Programminhalte über verschiedene Gruppen hinweg völlig standardisiert anzubieten, weil das bedeuten würde, auf Besonderheiten der jeweiligen Teilnehmenden nicht einzugehen. All dies erschwert (quantitative) Forschung sowohl zur Wirksamkeit als auch zu den Wirkungsmechanismen.

Dem beschriebenen Forschungsdesiderat wird im vorliegenden Beitrag begegnet. Im Rahmen einer abenteuer- und erlebnispädagogischen Jugendfreizeit werden das Selbstwertgefühl, die Selbstwirksamkeit und das emotionale Wohlbefinden bei teilnehmenden Heranwachsenden betrachtet, wobei die Frage im Fokus steht, wie Veränderungen im Verlauf des Programms zu Stande kommen: Welche spezifischen Erfahrungen sind es, die mit größeren (oder kleineren) Veränderungen bei den Jugendlichen zusammenhängen? Anknüpfend an erlebnispädagogische Theoriebildung gehen wir davon aus, dass 1) die Neuartigkeit der Erfahrungen, 2) die erfolgreiche Selbstüberwindung, 3) das persönliche Kompetenzerleben und 4) die Qualität der Einbindung in eine Gruppe zentrale Erfolgsbedingungen in der Abenteuer- und Erlebnispädagogik darstellen können (vgl. dazu Walsh und Golins 1976; Luckner und Nadler 1997; McKenzie 2003; Deane und Harré 2013). Entsprechend prüft der Beitrag, welche dieser Bedingungen mit Veränderungen bei den Teilnehmenden kor- 
reliert sind, um empirische Anhaltspunkte zu erhalten, auf welche Art und Weise eine Wirkung zu Stande kommt.

\section{Theoretischer Rahmen, Forschungsstand und Hypothesen}

\subsection{Erfahrungslernen als Ausgangspunkt erlebnispädagogischer Theoriebildung}

In der erlebnispädagogischen Literatur rekurrieren Wirksamkeitsbegründungen häufig auf die grundlegenden Überlegungen zum Erfahrungslernen von Kolb (1984). Für Kolb sind Lernprozesse und hiermit verknüpfte Erkenntnisse immer erfahrungsbegründet. In seinem Modell beschreibt er Lernen deshalb als einen aus vier Phasen zusammengesetzten zyklischen Prozess: 1) Konkrete Beobachtungen, Wahrnehmungen und Erlebnisse werden 2) reflexiv verarbeitet und 3) begrifflich-konzeptionell in bestehendes Wissen integriert, welches 4) in neuen Situationen angewendet wird, die zu weiteren Erlebnissen führen können und den Zyklus des Erfahrungslernens von Neuem in Gang setzen. Diese Überlegungen sind leicht auf abenteuer- und erlebnispädagogische Prozesse übertragbar: So stehen im Zentrum entsprechender Programme vor allem Erlebnisse, also Ereignisse, die durch ihre besondere Qualität außerhalb der „,normalen“ und bekannten Wahrnehmungs-, Deutungs- und Handlungsroutinen eines Akteurs liegen. Diese Erlebnisse werden durch Rückblick und Reflexion in Erfahrungen und Erkenntnisse transformiert, die im Idealfall zu einem veränderten Selbst- und/oder Weltbild führen und auf neue Situationen transferiert werden können. So gesehen sind Erlebnisse nicht nur Ausgangspunkt eines Lernprozesses im engeren Sinne, sondern vielmehr Anstoß für eine ganzheitliche Persönlichkeitsbildung (Fischer und Ziegenspeck 2008, S. 24 ff.). Unmittelbar einleuchtend ist aber auch, dass nicht alles, was Menschen erleben, einen signifikanten Lern- oder Bildungsprozess nach sich zieht, sondern dies nur für Erfahrungen gilt, die sich durch bestimmte Qualitäten auszeichnen.

\subsection{Welche Qualitäten haben wirksame Erfahrungen?}

$\mathrm{Zu}$ den vermutlich am häufigsten zitierten Erklärungsmodellen zur Wirkungsweise erlebnispädagogischer Programme gehören u.a. das „Outward Bound Process Model“" von Walsh und Golins (1976) und das Lernzonenmodell von Luckner und Nadler (1997). Ihre grundlegenden Überlegungen wurden später u. a. von McKenzie (2003), Sibthorp (2003) sowie Deane und Harré (2013) aufgegriffen, ergänzt, erweitert und zum Teil auch kritisiert. Im Kern wird aber in allen Beiträgen argumentiert, dass insbesondere die für erlebnispädagogische Interventionen typische neuartige, natürliche Umgebung, die zumeist herausfordernde Problembewältigung, welche mit Anstrengung und Überwindung verbunden ist, die Kompetenzerlebnisse, die mit dem Lösen einer gestellten Aufgabe einhergehen, sowie das Gemeinschaftserleben in einer sich unterstützenden Kleingruppe die wesentlichen Auslöser für Veränderungsprozesse darstellen. Die zentralen Faktoren in diesen Ansätzen stellen folglich 1) Neuartigkeit, 2) Herausforderung, 3) Kompetenzerleben und 4) soziale 
Einbindung dar. Wir begründen im Folgenden die Bedeutung dieser Einflussfaktoren genauer.

Neuartigkeit: Ein Ausgangspunkt liegt in der Annahme, dass vor allem eine neue, bisher fremde Umgebung bzw. eine unvertraute Aktivität neuartige Erfahrungsmöglichkeiten eröffnet. Die Umgebung und Aktivitäten sollen einen Kontrast zum Gewohnten und Bekannten darstellen und so einerseits eine veränderte Selbstwahrnehmung und andererseits neue Sichtweisen auf das Bekannte ermöglichen. Darüber hinaus werden eine unbekannte Umgebung bzw. eine unvertraute Aktivität auch als aktivierende Aspekte beschrieben, die Aufmerksamkeit und Achtsamkeit erhöhen. Erlebnispädagogische Programme zeichnen sich also dadurch aus, dass sie aufgrund der Verlagerung in ,,außergewöhnliche“ Kontexte und des Durchführens von neuen Aktivitäten - z.B. Camping in der Wildnis, Wandern im Hochgebirge, Segeln auf offener See - ein Aufbrechen alltäglicher Wahrnehmungs-, Deutungs- und Handlungsroutinen forcieren. Zahlreiche theoretische Ausarbeitungen stützen diese Annahme und betonen die Bedeutsamkeit der neuartigen, fremden Umgebung und Aktivität (Kimball und Bacon 1993; Nadler 1993; Luckner und Nadler 1997; Goldenberg et al. 2005). Und umgekehrt: Wer sich immer nur bekannten und bewährten Situationen aussetzt, wird in der Regel wenig Neues über sich erfahren, sondern einmal etablierte Selbst- und Weltkonzepte immer wieder bestätigen.

Herausforderung: Ein weiterer zentraler Bestandteil erlebnispädagogischer Maßnahmen sind Aktivitäten, die von den Teilnehmenden als herausfordernd wahrgenommen werden (Panicucci 2007; Brown 2008; Stremba und Bisson 2009). Diese Herausforderungen können im physischen, psychischen oder auch sozialen Bereich liegen (Heckmair und Michl 2008). Dabei wird in der erlebnispädagogischen Theoriediskussion stets hervorgehoben, dass die Teilnehmenden einerseits mit Herausforderungen konfrontiert, andererseits jedoch nicht überfordert werden sollen (Hattie et al. 1997; Hopkins und Putnam 1997). Am deutlichsten haben Luckner und Nadler (1997) diesen Aspekt herausgearbeitet: Sie beschreiben, dass sich die Teilnehmenden durch die Konfrontation mit unbekannten Situationen und herausfordernden Aktivitäten aus ihrer „comfort zone“ herausbegeben müssen und in eine Lernzone, die ,groan zone“ oder „,growth zone“, eintreten. Der Herausforderungscharakter einer Aktivität kann sich dabei aus verschiedenen Aspekten ergeben: einer physischen Anstrengung (z. B. bei einer schwierigen Bergwanderung), dem Verzicht auf Komfort (z. B. beim Übernachten in der Wildnis) oder dem Überwinden von Ängsten (z. B. beim Klettern in großer Höhe). Solchen Momenten der Überwindung und der Grenzerfahrung gehen Zweifel und Unsicherheit voraus: Dean und Harré (2013) sprechen hier von einem ,psychological disequilibrium“. D. h., ein bestimmtes Maß an Risiko, Zweifel und Unsicherheit ist geradezu konstitutiv für eine Erfahrung, die rückblickend als „Abenteuer“ klassifiziert und subjektiv mit Bedeutung versehen wird. Gleichwohl liegt die ,groan zone“, in der die beschriebenen Grenzerfahrungen möglich sind, auf einem schmalen Grat. Übersteigt die Anforderung die Fähigkeiten einer Person deutlich, gerät diese in die ,panic zone“ und aus dem erhofften Abenteuer wird ein „misadventure“ (Nichols 2000). Entsprechend enthalten viele Abenteuerprogramme Bausteine in verschiedenen Schwierigkeitsgraden. 
Kompetenzerleben: Erst unter den eben beschriebenen Voraussetzungen eines anfänglichen Zweifels, von Unsicherheit und Selbstüberwindung können Herausforderungen, wenn sie eben doch gemeistert und bewältigt wurden, subjektiv als bedeutsame Kompetenzerlebnisse reflektiert werden (Walsh und Golins 1976; McKenzie 2000). Einige Studien (u.a. McKenzie 2003; Cordle et al. 2016) haben gezeigt, dass die Kombination aus Herausforderung, Bewältigung und Kompetenzerleben erwünschte Veränderungen, z. B. auf Selbstkonzept und Selbstwirksamkeit, auslöst. Das persönliche Kompetenzerleben - im Modell von Walsh und Golins (1976) als „Mastery“ bezeichnet - kann somit als weitere Erfolgsbedingung betrachtet werden. Dabei kann man davon ausgehen, dass ein Kompetenzerlebnis umso tiefgreifender und prägender ist, wenn die durchgeführte Aktivität neuartig und herausfordernd war bzw. sich eine Person das Meistern vorher selbst gar nicht zugetraut hätte. In gewisser Weise sind die zuvor genannten Punkte also implizit mitgedacht, denn es geht nicht um beliebige Kompetenzerlebnisse, sondern solche, die sich bei neuen Aktivitäten und beim Überwinden von Widerständen einstellen. Die Aufgabe eines effektiven Programms wäre es also, Herausforderungen so zu dosieren, dass ein Meistern nicht sicher, aber doch wahrscheinlich ist. Weiterhin wird in vorliegenden Beiträgen (u.a. McKenzie 2000) argumentiert, dass Aktivitäten möglichst ganzheitlich ausgerichtet sein sollen, d.h. mentale, emotionale und physische Kapazitäten beanspruchen sollen, damit die Bewältigungserfahrung auf mehrere Bereiche bezogen werden kann. Was Teilnehmende ggf. auch aus Erfahrungen des Scheiterns lernen, ist so gut wie nicht erforscht. Zumindest im Hinblick auf Selbstwirksamkeit dürften sich aber aus gescheiterten Bemühungen sicher keine positiven Veränderungen ergeben.

Soziale Einbindung: In der Theoriediskussion wird darüber hinaus immer wieder auf eine optimale Gruppengröße (7 bis 15 Personen) hingewiesen. Die Vorteile der Kleingruppe werden darin gesehen, dass sich eine Cliquenbildung innerhalb der Gruppe so verhindern lässt (Walsh und Golins 1976; McKenzie 2000). Ferner besteht die Annahme, dass die soziale Einbindung Einzelner und das „Wir-Gefühl“ in der Gruppe die Effekte erlebnispädagogischer Programme beeinflussen (Martin und Leberman 2005; Cook 2008; D’Amato und Krasny 2011). In kleineren Gruppen können sich die Teilnehmenden in der Regel schneller kennenlernen, ihre Stärken und Schwächen wechselseitig einschätzen und deshalb beim Lösen von Aufgaben und Problemen effektiver zusammenarbeiten. Auf diese Weise kann sich eine Person eher als in großen Gruppen als wichtiges, Verantwortung tragendes Mitglied der Gruppe erleben; d.h. der eigene Beitrag zum Gruppenerfolg ist nicht marginal, sondern wird als bedeutsam erlebt. Einige Studien stellen zudem fest, dass enge Gruppenbindungen in erlebnispädagogischen Projekten oft entstehen und der $\mathrm{Zu}-$ sammenhalt bzw. die Hilfsbereitschaft meist sehr hoch ist (Duerden und Witt 2010; Draper et al. 2011).

Bei den angesprochenen Aspekten handelt es sich um vier Kernelemente pädagogischer Programme, die darauf abzielen durch „Abenteuer“ und „Erlebnis“ individuelle Entwicklungsprozesse in Gang zu setzen. Inwiefern dies gelingt, ob also die angebotenen Aktivitäten als neu und herausfordernd erlebt werden, sich die Teilnehmenden wirklich als kompetent und als wichtigen Teil der Gruppe erleben, ist aber keineswegs gewiss. Vielmehr werden die Programminhalte individuell verschieden 
erlebt und verarbeitet. Diese individuellen Erfahrungen sollten theoretisch erklären können, warum ein Programm unterschiedlich wirkt, z. B. bei einigen Personen positive Veränderungen auslöst, bei anderen hingegen nicht.

\subsection{Forschungsstand zur Wirkungsweise erlebnispädagogischer Programme}

Die meisten der vorliegenden Evaluationsstudien beschreiben die Wirksamkeit erlebnispädagogischer Programme ausschließlich in Form von Vorher/Nachher-Vergleichen. Dabei bleiben die Faktoren, die Veränderungen auslösen, häufig unerforscht. Die Intervention selbst ist damit eine Black Box, in der ,irgendetwas“ erfolgt ist, das (vermutlich) zu den beobachteten oder gemessenen Effekten geführt hat (Klawe 2006). Obwohl Hattie et al. (1997) oder Priest (1999) diesbezüglich bereits vor 20 Jahren auf ein Forschungsdefizit hinwiesen, hat sich bislang erst wenig getan, so dass neuere Beiträge (z.B. Lakemann 2017; Fengler 2017; Heekerens 2018) noch immer auf die gleiche Forschungslücke verweisen und fordern, den Wirkmechanismen - nun endlich - auf den Grund zu gehen.

Gleichwohl liefern einige Studien erste Einblicke in potenzielle Wirkungsweisen erlebnispädagogischer Interventionen. Sie elaborieren konkrete Erfolgsbedingungen für diese Programme, weisen zugleich aber einige methodische Defizite auf. Qualitativ angelegte Studien (u.a. McDonald et al. 2009; D’Amato und Krasny 2011; Draper et al. 2011; Hinds 2011) stützen sich auf umfangreiches Interviewmaterial mit Teilnehmenden und arbeiten hieran jene Erfahrungen und Bedingungen heraus, die subjektiv als besonders bedeutsam und als Auslöser von Transformationsprozessen reflektiert werden. Verwiesen wird in diesen Studien z.B. auf tiefgreifende Naturerlebnisse, die die Erhabenheit der Landschaft, die Einsamkeit und Stille in der Wildnis oder das Gefühl der Verbundenheit mit der Natur beschreiben; auf Momente, in denen Herausforderungen unter großen Anstrengungen gemeistert werden konnten; aber z.B. auch auf die Wertschätzung und Akzeptanz, die die Teilnehmenden in der Gruppe erfahren konnten. Das Aufzeigen subjektiv bedeutsamer Erlebensmomente ist aber nicht identisch mit Wirkmechanismen. Hierfür müssten Erfahrungen genauer und systematisch mit individuellen Veränderungen in Relation gebracht werden.

Die Verbindung von Erfahrungen mit Wirkungen versuchen andere Studien, die halbstrukturierte Fragebogen- und Interviewdaten erhoben haben, explizit zu machen (McKenzie 2003; Goldenberg et al. 2005; Paisley et al. 2008; Sibthorp et al. 2011). In diesen Arbeiten werden Teilnehmende nach dem Abschluss eines Programms nach ihrer Meinung zur Wirksamkeit gefragt sowie nach den Bedingungen, die ihrer Ansicht nach die Wirkung ausgelöst haben. Die Ergebnisse zeigen z. B., dass die Qualität der Anleitung durch die Betreuungsperson, das individuelle Meistern von Aufgaben, der Grad der physischen Herausforderung oder das Ausprobieren von neuen Aktivitäten von den Teilnehmenden als wichtige Aspekte gedeutet werden, die mit dem Erreichen der Zielsetzungen der Programme eng verkoppelt sind. Auch wenn sich diese Beiträge explizit mit der Frage des, impact that various course components had on several course outcomes“ (McKenzie 2003, S. 10) befassen möchten, so ist kritisch zu sehen, dass es sich um retrospektive Selbsteinschätzungen 
bzw. „Laientheorien“ darüber handelt, welche Bedingung welche Wirkung ausgelöst hat.

Sibthorp et al. (2007) haben noch etwas genauer nach Einflüssen geschaut, von denen die Wirkung eines abenteuer- und erlebnispädagogischen Programms abhängt. Sie kommen für fast alle Wirkungen (wie z. B. auf Kommunikations-, Sozial- und Führungskompetenzen) zu dem Ergebnis, dass Personen mit nur geringen Vorerfahrungen stärker von einem Programm profitieren als Personen mit umfangreicheren Vorerfahrungen. Das ist plausibel und entspricht der Überlegung, dass die Neuartigkeit einer Aktivität für deren Wirkung von Bedeutung ist. Gleichwohl handelt es sich hier erstens nicht um Prozess-Ergebnis-Forschung, weil Programmprozesse als solche gar nicht berücksichtigt werden. Und zweitens besteht ein methodisches Problem der Studie darin, dass es nur einen Zeitpunkt der Datenerhebung gab, und zwar nach Abschluss des Programms, an dem Einschätzungen über die Kompetenzen vom Beginn des Programms retrospektiv erhoben wurden. Die Autoren sprechen von einem ,retrospective pretest“" (Sibthorp et al. 2007), de facto handelt es sich aber um ein Querschnittdesign, mit dem Wirkungsweisen nicht aufzuklären sind.

Retrospektiv angelegte Studien, wo die Teilnehmenden am Ende des Programms über Erfahrungen und Wirksamkeit reflektieren, halten wir grundsätzlich für unzureichend für eine Prozess-Ergebnis-Forschung. Nur sehr wenige Studien haben die Wirkungsweisen bzw. die Erfolgsbedingungen erlebnispädagogischer Programme aber methodisch überzeugend erforscht. Zwei neuseeländische Evaluationen von erlebnispädagogischen Segelreisen (Scarf et al. 2018; Arahanga-Doyle et al. 2019) zeigen, dass eine Vielzahl an positiven Veränderungen mit dem wahrgenommenen Zusammengehörigkeitsgefühl und der Unterstützung innerhalb der Segelgruppe korreliert sind. Beide Studien nutzen dabei - ähnlich wie wir im Folgenden - ein PrätestPosttest-Design mit zwei Erhebungszeitpunkten am ersten und letzten Tag des Programms. Ob bzw. inwieweit aber die Befunde vom Segeln auch auf andere Formate und Inhalte der Abenteuer- und Erlebnispädagogik übertragen werden können, ist eine ungeklärte Frage. Hier setzt die vorliegende Studie an.

\subsection{Hypothesen}

Aufgrund der dargestellten theoretischen Überlegungen und der Ergebnisse der oben zitierten Forschung lässt sich davon ausgehen, dass die Neuartigkeit der Erfahrungen, die erfolgreiche Selbstüberwindung, das persönliche Kompetenzerleben und die Qualität der Einbindung in eine Gruppe als zentrale Erfolgsbedingungen erlebnispädagogischer Maßnahmen angesehen werden können. Basierend auf einer Stichprobe von Jugendlichen und jungen Erwachsenen, die ein zehntägiges abenteuer- und erlebnispädagogisches Ferienprogramm absolviert haben, werden diese Einflussfaktoren mit individuellen Veränderungen in Beziehung gesetzt. Drei Wirkungen - die im Grunde bereits gut erforscht sind - stehen dabei im Fokus: Selbstwirksamkeit, Selbstvertrauen und emotionales Wohlbefinden. Wir prüfen im Folgenden, ob die Stärke der Veränderungen im Verlauf des Programms systematisch mit einzelnen Erlebnisqualitäten zusammenhängen. Konkret gehen wir von vier Hypothesen aus: 
Hypothese 1: Im Vergleich zum Beginn des Programms fallen Selbstwirksamkeit, Selbstwertschätzung und affektives Wohlbefinden am Ende umso positiver aus, je mehr neuartige Aktivitäten die Teilnehmenden im Verlauf des Programms absolviert haben.

Hypothese 2: Im Vergleich zum Beginn des Programms fallen Selbstwirksamkeit, Selbstwertschätzung und affektives Wohlbefinden am Ende umso positiver aus, je mehr Aktivitäten die Teilnehmenden absolvierten, bei denen sie vorher Zweifel hatten, diese erfolgreich bewältigen zu können.

Hypothese 3: Im Vergleich zum Beginn des Programms fallen Selbstwirksamkeit, Selbstwertschätzung und affektives Wohlbefinden am Ende umso positiver aus, je stärker sich die Teilnehmenden bei ihren Aktivitäten als kompetent erlebten.

Hypothese 4: Im Vergleich zum Beginn des Programms fallen Selbstwirksamkeit, Selbstwertschätzung und affektives Wohlbefinden am Ende umso positiver aus, je mehr sich die Teilnehmenden bei den Aktivitäten in die Gruppe eingebunden fühlten.

\section{Methodik}

\subsection{Die Untersuchungsgruppe}

Zur Überprüfung der Annahmen wurden Jugendliche und junge Erwachsene im Alter zwischen 13 und 20 Jahren untersucht, die zwischen Mai und Juli 2016 an einer zehntägigen abenteuer- und erlebnispädagogischen Jugendfreizeit (im Rahmen einer Schulfreizeit oder eines Jugendcamps) in den französischen Cevennen teilnahmen. Die Größe der jeweiligen Gruppenfreizeiten lag zwischen 15 und 20 Teilnehmenden. Das Sample umfasst ausschließlich deutschsprachige Gruppen, die an der Freizeit während der gesamten zehn Tage teilnahmen. Die ausgewählten Gruppen konnten nahezu vollständig befragt werden, Ausnahmen stellen lediglich wenige Heranwachsende dar, die an den Aktivitäten aufgrund von Verletzungen oder Erkrankungen nicht in vollem Umfang teilnehmen konnten sowie zwei Jugendliche, die keine Erlaubnis der Eltern zur Teilnahme an der Studie vorlegen konnten. Darüber hinaus erfolgte die Teilnahme an der Studie selbstverständlich freiwillig.

Insgesamt wurden 108 Teilnehmende am Tag der Ankunft und am Tag der Abreise mit Hilfe eines standardisierten Fragebogens befragt. Um die Daten der ersten Erhebung $(t 1=$ Anreise $)$ mit denen der zweiten Befragung $(t 2=$ Abreise $)$ verknüpfen zu können und zugleich ein hohes Maß an Anonymität beim Ausfüllen gewährleisten zu können, wurden persönlich generierte Identifikationscodes (bestehend aus vier Ziffern) eingesetzt. Bei der späteren Auswertung konnten 32 Fragebögen (29,6\%) aufgrund abweichender Identifikationscodes nicht eindeutig zugeordnet werden, was der bei diesem Verfahren üblichen Verlustrate entspricht (Schnell et al. 2010). Insgesamt stützen wir uns deshalb im Folgenden auf die vollständigen, zwei Erhebungszeitpunkte umfassenden Angaben von 76 Teilnehmenden, hierunter 28 weibliche und 48 männliche Personen. 


\subsection{Das abenteuer- und erlebnispädagogische Programm}

Die Cevennen, der südöstliche Teil des französischen Zentralmassivs, ist geprägt von engen, steilen Schluchten sowie Hochebenen und bekannt für die vielfältigen Möglichkeiten zum Klettern, Bergwandern, Kanu- und Wildwasserfahren. Während der gesamten Programmdauer lebten die Teilnehmenden in einem Basislager auf einem nichtöffentlichen Campingplatz inmitten der Natur und übernachteten in Zelten. Für die Organisation des Camps waren die jeweiligen Gruppen mitverantwortlich und übernahmen z.B. die Essenszubereitung. Auf dem Campingplatz und dem umliegenden Areal gab es keinen Zugang zum Internet, keine Möglichkeit fernzusehen und keinen Handyempfang.

Jugendliche und junge Erwachsene stellen die Zielgruppe dieser Freizeit dar, deren Ziele vor allem Persönlichkeitsentwicklung und Gruppenzusammenhalt adressieren. Vor dem Hintergrund dieser Zielsetzung und der gleichzeitigen Freiwilligkeit an der Teilnahme lässt sich die Intervention als ein pädagogisch gerahmtes Freizeitprogramm beschreiben. Es fand in allen Gruppen in einem ähnlichen Ablauf und unter Anleitung des gleichen Personals statt.

Im Rahmen der Ferienfreizeit wurden unter Aufsicht und Anleitung von abenteuer- und erlebnispädagogisch geschultem und in den jeweiligen Handlungsfeldern qualifiziertem Personal die folgenden Aktivitäten angeboten:

1. eine Wildwasser-Kanufahrt, bei der die Teilnehmenden in Gruppen mit einem Kanu auf einem Fluss fahren und dabei Stromschnellen und Hindernisse bewältigen müssen;

2. Abseilen vom Fels, wobei die Teilnehmenden sich sowohl aus über 40 Metern von einer überhängen Felswand als auch in eine Höhle hinein abseilen;

3. eine Höhlenexpedition, bei der die Teilnehmenden ohne technische Hilfsmittel eine enge und weit verzweigte Höhle erkunden;

4. das Absolvieren eines Klettersteigs, d.h. einer mit Stahlgeländern gesicherten Kletterroute, die u. a. ausgesetzte Kletterpassagen in großer Höhe beinhaltet;

5. Canyoning, d.h. das Durchwandern eines steilen Flussbetts, wobei Schwimmpassagen, das Springen von Klippen und das Abseilen an einem Wasserfall zu bewältigen sind;

6. Klettern am Fels in unterschiedlichen Schwierigkeitsgraden.

Die aufgeführten Aktivitäten, die als wechselnde Tagesangebote absolviert wurden, konnten frei gewählt werden, so dass jeden Tag neue Kleingruppen in etwas unterschiedlicher Zusammensetzung gebildet wurden. Die Jugendlichen erhielten über die jeweilige Aktivität zunächst alle vorliegenden Informationen, so dass sie die Aktivität und damit den Grad der Herausforderung selbst wählen konnten (,Challenge by Choice“). Dabei bestand grundsätzlich auch die Möglichkeit, eine Aktivität mehrmals zu wählen. Alle Aktivitäten folgten darüber hinaus den Gestaltungsparadigmen der Abenteuerpädagogik: Die Durchführung erfolgte ausschließlich in der Natur; die Gruppengröße war auf 10 Personen begrenzt; alle Aktivitäten beinhalteten körperliche, z.T. auch psychische Herausforderungen; die bei den Aktivitäten ggf. entstehenden sozialen Konflikte mussten primär von der Gruppe selbst gelöst 
bzw. bewältigt werden; nach den Aktivitäten fand eine angeleitete Reflexionsphase statt, in der das Erlebte gemeinschaftlich thematisiert wurde.

\subsection{Indikatoren}

Die folgenden Analysen beziehen sich auf drei abhängige Variablen, deren Veränderungen im Verlauf des Programms betrachtet werden.

1. Allgemeine Selbstwirksamkeitserwartungen wurden mit der gleichnamigen Skala von Schwarzer und Jerusalem (1999) erhoben. Die Befragten konnten ihre Zustimmung zu Aussagen wie z. B. „Wenn sich Widerstände auftun, finde ich Mittel und Wege, mich durchzusetzen“ auf einer vierstufigen Skala von 1=,stimmt nicht“ bis $4=$,stimmt genau“ angeben. Die Skala besteht aus zehn Items und besitzt eine gute Reliabilität (Cronbachs $\alpha_{\mathrm{t} 1}=0,84$; Cronbachs $\alpha_{\mathrm{t} 2}=0,90$ ).

2. Das Selbstwertgefühl wurde als Kurzskala mit Items der deutschsprachigen Version der Rosenberg Self-Esteem Scale erhoben (Rosenberg 1965; Ferring und Fillip 1996). Die Skala enthält vier Items (z. B. „Ich habe eine positive Einstellung zu mir selbst gefunden“) und entspricht der von Tambs und Røysamb (2014) geprüften Kurzversion. Die Befragten konnten auch hier ihre Zustimmung auf einer Likert-Skala von $1=$,,lehne voll und ganz ab“ bis $4=$,,stimme voll und ganz zu“ angeben. Die Skala besitzt eine gute Reliabilität (Cronbachs $\alpha_{t 1}=0,73$; Cronbachs $\left.\alpha_{\mathrm{t} 2}=0,80\right)$.

3. Das affektive Wohlbefinden wird in der Zufriedenheitsforschung üblicherweise als Differenzwert aus positiven und negativen Emotionen operationalisiert (,hedonic balance“, vgl. Bradburn 2015). Die Jugendlichen wurden danach gefragt, wie häufig sie positive Emotionen (sich ,glücklich“, „,voller Freude“, ,,voller Energie“ fühlen, „das Leben genießen“) und negative Emotionen (sich „traurig“ und „einsam“ fühlen, sich „Sorgen machen“, sich „zu nichts aufraffen“ können) in der letzten Woche erlebt haben. Die Antwortvorgaben reichten hier von $1=$,fast nie“" bis 6=,fast immer“. Die Items sind dem Kernmodul „Wellbeing“ des European Social Survey entnommen. Anschließend wurde der Mittelwert der negativen Emotionen vom Mittelwert der positiven Emotionen abgezogen $\left(M_{\mathrm{pos}}-M_{\text {neg }}\right)$. D.h., Werte größer Null zeigen an, dass eine Person mehr positive als negative Emotionen erlebt hat und Werte kleiner Null signalisieren das Gegenteil, also die relative Dominanz negativer Affekte.

Darüber hinaus wurden die vier ausgewählten möglichen Einflussfaktoren des Programms operationalisiert, die Veränderungen bewirken könnten. Dabei wurde in einem ersten Schritt erfragt, welche der sechs oben genannten Aktivitäten eine Person im Verlauf der 10 Tage ausgeübt hat. Im zweiten Schritt wurden dann zu jeder Aktivität, die ausgeübt wurde, weitere Angaben erhoben, z. B. ob die Aktivität zum ersten Mal ausgeübt wurde.

1. Der Aspekt der Neuartigkeit wurde mit der Anzahl der Aktivitäten gemessen, die ein Jugendlicher zum ersten Mal ausgeübt hat. Die Variable kann Ausprägungen zwischen 0 (keine neue Aktivität) und 6 (sechs neue Aktivitäten) annehmen $(M=2,18 ; S D=1,55)$. 
2. Der Grad der Selbstüberwindung wurde mit der Frage „Ich habe bei dieser Aktivität etwas geschafft, das ich mir vorher nicht zugetraut hätte“ erhoben. Die Befragten konnten ihre Antwort zwischen 1=,trifft gar nicht zu“ und 5=,,trifft voll und ganz zu“ abstufen. Berechnet wurde der Mittelwert aus den Angaben zu allen von der Person im Lauf der zehn Tage absolvierten Aktivitäten $(M=3,09 ; S D=1,27)$.

3. Das Kompetenzerleben wurde mit der Frage operationalisiert „Ich habe mich bei allem, was ich bei dieser Aktivität tun musste, kompetent gefühlt“. Die Befragten konnten ihre Antwort wieder von $1=$,trifft gar nicht zu“ bis 5=,trifft voll und ganz zu“" abstufen. Wiederum wurde der Mittelwert aller Aktivitäten berechnet $(M=3,99 ; S D=0,80)$.

4. Um die Gruppeneinbindung bei den ausgeübten Aktivitäten zu ermitteln, konnte die Zustimmung zur Aussage ,Ich habe mich mit den anderen als ein Team gefühlt" auf der gleichen fünfstufigen Antwortskala (s. oben) abgestuft werden. Aus den gültigen Angaben wurde auch hier der Mittelwert berechnet $(M=3,93$; $S D=0,83)$.

Diese vier Einflussfaktoren sind punktuell miteinander korreliert. So findet sich eine positive Korrelation zwischen „Neuartigkeit“ und „Selbstüberwindung“ ( $r=0,41$; $p<0,01)$ sowie zwischen „Kompetenzerleben“ und „Gruppeneinbindung“ ( $r=0,38$; $p<0,01)$. Alle weiteren Korrelationen zwischen den Prädiktoren sind von geringem Betrag und nicht signifikant.

Zusätzlich werden in den Modellen drei Kontrollvariablen berücksichtigt: das Alter in Jahren $(M=17,8 ; S D=1,26 ; \min =13 ; \max =20)$, das Geschlecht $(1=$ weiblich, $0=$ männlich) und ein Indikator für den materiellen Wohlstand der Familie. Letzterer wurde aus einer Liste von Wohlstandsgütern berechnet, die sich auf das Elternhaus beziehen (,Gibt es bei dir zu Hause ...a) einen Garten, b) ein Gästezimmer, c) eine Geschirrspülmaschine, d) Kunstwerke, e) mehrere Badezimmer, f) größere Fitnessgeräte, g) einen Blu-Ray Player"). Aus diesen Angaben wurde ein additiver Index berechnet $(M=5,04 ; S D=1,49 ; \min =0 ; \max =7)$.

\subsection{Analyseverfahren}

Mit Hilfe des Prätest-Posttest-Designs mit zwei Messzeitpunkten können zunächst systematische Veränderungen in den drei abhängigen Variablen aufgezeigt werden. Diese werden mittels t-Tests für verbundene Stichproben geprüft. Um die Größenordnung der Veränderungen einschätzen zu können, werden Effektstärken $(d)$ berechnet, die sich aus dem Mittelwert der Veränderungen von $t 1 \mathrm{zu} t 2$ und der Standardabweichung dieser Veränderungswerte ergeben. Diese Tests geben zunächst Auskunft über durchschnittliche Veränderungen im Verlauf des Programms.

Die Frage der Wirkungsweise wird im Anschluss mit drei linearen Regressionsmodellen geprüft. In diesen Modellen werden die $t 2$-Werte der Selbstwirksamkeitserwartung, der Selbstwertschätzung und des affektiven Wohlbefindens als abhängige Variablen betrachtet. In einem ersten Schritt wird der systematische Einfluss der $t 1$-Werte jeder Variable auf die $t 2$-Werte berechnet. Die Residualvarianz - also die nicht durch die Ausgangswerte erklärbare Veränderung zu $t 2$ - lässt sich als Veränderungsvarianz interpretieren (Reinders 2006). Sie verdeutlicht, bei wel- 
chen Personen die Veränderung im Verlauf des Programms größer (bzw. kleiner) als erwartet ausgefallen ist. Im zweiten Schritt werden die vier diskutierten Einflussfaktoren (Neuigkeit, Selbstüberwindung, Kompetenzerleben, Gemeinschaftsbindung) als Prädiktoren in das Modell einbezogen. Dabei wird zunächst der Effekt jedes Einflussfaktors einzeln geschätzt und danach ein Gesamtmodell berechnet, das alle Prädiktoren gemeinsam einbezieht. In allen Regressionsmodellen werden zudem Alter, Geschlecht und der materielle Wohlstand des Elternhauses als Kontrollvariablen berücksichtigt. Der hier verfolgte Ansatz wird in der Literatur u. a. unter den Stichworten „lagged regression“, ,,autoregression model“ oder ,,residualized change“ diskutiert. Im Vergleich zu anderen Ansätzen, die auf individuellen Differenzen von $t 1$ - und $t 2$-Werten basieren, zeichnet er sich u. a. dadurch aus, dass die Größenordnung von individuellen Veränderungen nicht so stark von der Höhe der individuellen Ausgangswerte beeinflusst wird (vgl. Newsom 2012).

\section{Befunde}

\subsection{Veränderungen im Verlauf des Programms}

Es zeigen sich für die Jugendlichen insgesamt signifikante und im Hinblick auf die Stärke beachtliche Veränderungen von $t 1 \mathrm{zu} t 2$ auf den hier betrachteten drei Kriterien (Tab. 1): Die Jugendlichen geben am Ende der Ferienfreizeit eine höhere Selbstwirksamkeitserwartung $(d=0,33)$, eine höhere Selbstwertschätzung $(d=0,46)$ und ein positiveres Wohlbefinden an $(d=0,94)$. Die Effektstärken liegen im mittleren bis hohen Bereich; sie entsprechen den aus Überblicksarbeiten bekannten Befunden (Hattie et al. 1997; Gillis und Speelman 2008).

\subsection{Wirkungsweisen des Programms}

Zur Beantwortung der zentralen Forschungsfrage des Beitrags nach den Wirkungsweisen prüfen wir vier Einflussfaktoren (Neuartigkeit, Selbstüberwindung, Kompetenzerleben, Gemeinschaftsbindung), deren Relevanz wir anfangs begründet haben. Die entsprechenden Regressionsmodelle betrachten Selbstwirksamkeit, Selbstwert und emotionales Wohlbefinden als abhängige Variablen. Die Modelle schätzen zunächst das Niveau der abhängigen Variablen zum Zeitpunkt $t 2$ unter Kontrolle des Ausgangsniveaus zum Zeitpunkt $t 1$ (Modell I). Die hierdurch nicht erklärte Varianz wird anschließend auf die im Programm gemachten Erfahrungen zurückgeführt: die vier Prädiktoren werden dabei erst einzeln (Modelle II-V) und dann gemeinsam

Tab. 1 Veränderungen von Selbstwirksamkeit, Selbstwert und emotionalem Wohlbefinden im Verlauf des zehntägigen Abenteuer- und Erlebnisprogramms

\begin{tabular}{lllllll}
\hline & \multicolumn{7}{l}{ Mittelwertvergleich $(t 1$ vs. $t 2)$} & & \\
& $t 1$ & $t 2$ & Diff & SD diff & $d$ & $p$ \\
\hline Selbstwirksamkeit & 2,87 & 3,00 & 0,13 & 0,40 & 0,33 & 0,009 \\
Selbstwert & 3,04 & 3,26 & 0,22 & 0,48 & 0,46 & $<0,001$ \\
Affektives Wohlbefinden & 1,11 & 2,80 & 1,69 & 1,79 & 0,94 & $<0,001$ \\
\hline
\end{tabular}


Tab. 2 Lineare Regressionsmodelle zur Vorhersage der Selbstwirksamkeitserwartung am Ende des Programms ( $t 2)$ unter Kontrolle der Ausgangswerte $(t 1)$

\begin{tabular}{|c|c|c|c|c|c|c|c|}
\hline & \multicolumn{7}{|c|}{ Selbstwirksamkeit $(t 2)$} \\
\hline & I & II & III & IV & $\mathrm{V}$ & VI & VI $\beta$ \\
\hline \multicolumn{8}{|l|}{ Ausgangswerte } \\
\hline $\begin{array}{l}\text { Selbstwirksamkeit } \\
(t 1)\end{array}$ & $0,82 * *$ & $0,88 * *$ & $0,91 * *$ & $0,78^{* *}$ & $0,90 * *$ & $0,76^{* *}$ & 0,64 \\
\hline \multicolumn{8}{|l|}{ Programmerfahrungen } \\
\hline Neuartigkeit & - & $0,07 *$ & - & - & - & $0,08^{*}$ & 0,23 \\
\hline Selbstüberwindung & - & - & 0,00 & - & - & $-0,04$ & $-0,09$ \\
\hline Kompetenzerleben & - & - & - & $0,16^{* *}$ & - & $0,13^{*}$ & 0,19 \\
\hline Gemeinschaftserleben & - & - & - & - & $0,08^{+}$ & 0,05 & 0,07 \\
\hline \multicolumn{8}{|l|}{ Kontrollvariablen } \\
\hline Alter & - & $-0,04$ & $-0,05$ & $-0,04$ & $-0,05$ & $-0,03$ & $-0,07$ \\
\hline Geschlecht & - & $-0,08$ & $-0,16$ & $-0,18^{+}$ & $-0,15$ & $-0,14$ & $-0,12$ \\
\hline Fam. Wohlstand & - & $-0,04$ & $-0,02$ & $-0,03$ & $-0,03$ & $-0,05$ & $-0,14$ \\
\hline $\mathrm{R}^{2}$ & 0,474 & 0,554 & 0,520 & 0,567 & 0,533 & 0,605 & \\
\hline $\begin{array}{l}\Delta \mathrm{R}^{2} \text { (im Vgl. zu } \\
\text { Modell I) }\end{array}$ & - & 0,080 & 0,046 & 0,093 & 0,059 & 0,131 & \\
\hline
\end{tabular}

Anmerkung: OLS Regression mit robusten Standardfehlern. Angegeben sind nicht-standardisierte Koeffizienten (b) sowie für Modell VI auch standardisierte Koeffizienten ( $\beta$ ). Signifikanz: ${ }^{+} p<0,10,{ }^{*} p<0,05$, $* * p<0,01$, im Fall der Variablen im Block „Programmerfahrungen“ basierend auf einseitigen Tests

(Modell VI) in der Regressionsschätzung berücksichtigt. Zudem ist die Veränderung im $\mathrm{R}^{2}$ im Vergleich zu Modell 1 ausgewiesen, um zu verdeutlichen, welchen Einfluss diese Erfahrungen auf die individuellen Unterschiede zum Zeitpunkt $t 2$ haben.

Eine positive Veränderung der Selbstwirksamkeit findet eher statt, je mehr neuartige Aktivitäten die Jugendlichen ausprobiert haben und je kompetenter sie sich dabei gefühlt haben (Tab. 2). Beide Einflussfaktoren sind in den Einzelmodellen, aber auch im Gesamtmodell signifikant. Wer also an vielen unterschiedlichen neuen Aktivitäten teilgenommen und sich dabei sehr kompetent erlebt hat, profitiert im Hinblick auf Selbstwirksamkeitserwartungen am meisten. Dabei scheint es egal zu sein, ob die Aktivität tatsächlich so herausfordernd ist, dass sie anfänglich Zweifel entstehen lässt und eine Selbstüberwindung erfordert. Der Aspekt der Selbstüberwindung ist im Modell nicht von Bedeutung. Das positive Gemeinschaftserleben bei einer Aktivität ist tendenziell auch mit einer geringen Steigerung der eigenen Selbstwirksamkeit assoziiert, der Effekt ist aber in der kleinen Stichprobe nicht statistisch signifikant. Insgesamt lassen sich die Unterschiede in der Selbstwirksamkeit am Ende des Programms zu $47 \%$ auf die Ausgangswerte und zu etwa 13\% auf die im Programm gemachten Erfahrungen zurückführen.

Die Veränderung der Selbstwertschätzung hängt besonders stark vom Kompetenzerleben bei der Aktivität ab (Tab. 3). Je kompetenter sich die Jugendlichen bei den ausgeübten Aktivitäten fühlten, desto positiver fällt die Veränderung im Selbstwert aus. Der Einfluss der Neuartigkeit einer Aktivität ist der Tendenz nach ebenfalls positiv $(p<0,10)$. Es ist also auch relevant, dass es sich dabei um neuartige Aktivitäten handelt, die die Jugendlichen erstmalig ausprobieren. Die Aspekte Selbstüberwin- 
Tab. 3 Lineare Regressionsmodelle zur Vorhersage der Selbstwertschätzung am Ende des Programms $(t 2)$ unter Kontrolle der Ausgangswerte $(t 1)$

\begin{tabular}{|c|c|c|c|c|c|c|c|}
\hline & \multicolumn{7}{|c|}{ Selbstwert $(t 2)$} \\
\hline & I & II & III & IV & $\mathrm{V}$ & VI & VI $\beta$ \\
\hline \multicolumn{8}{|l|}{ Ausgangswerte } \\
\hline Selbstwert $(t 1)$ & $0,70 * *$ & $0,74 * *$ & $0,73 * *$ & $0,60 * *$ & $0,69 * *$ & $0,58 * *$ & 0,56 \\
\hline \multicolumn{8}{|l|}{ Programmerfahrungen } \\
\hline Neuartigkeit & - & $0,08^{+}$ & - & - & - & $0,07^{+}$ & 0,14 \\
\hline Selbstüberwindung & - & - & $-0,01$ & - & - & $-0,05$ & $-0,08$ \\
\hline Kompetenzerleben & - & - & - & $0,32 * *$ & - & $0,28 * *$ & 0,28 \\
\hline Gemeinschaftserleben & - & - & - & - & 0,11 & 0,06 & 0,06 \\
\hline \multicolumn{8}{|l|}{ Kontrollvariablen } \\
\hline Alter & - & $-0,09$ & $-0,10$ & $-0,07$ & $-0,09$ & $-0,07$ & $-0,11$ \\
\hline Geschlecht & - & $-0,14$ & $-0,22$ & $-0,29^{*}$ & $-0,18$ & $-0,28^{+}$ & $-0,16$ \\
\hline Fam. Wohlstand & - & $-0,04$ & $-0,02$ & $-0,03$ & $-0,03$ & $-0,04$ & $-0,08$ \\
\hline $\mathrm{R}^{2}$ & 0,462 & 0,534 & 0,511 & 0,593 & 0,523 & 0,605 & \\
\hline $\begin{array}{l}\Delta \mathrm{R}^{2} \text { (im Vgl. zu } \\
\text { Modell I) }\end{array}$ & - & 0,072 & 0,049 & 0,131 & 0,061 & 0,143 & \\
\hline
\end{tabular}

Anmerkung: OLS Regression mit robusten Standardfehlern. Angegeben sind nicht-standardisierte Koeffizienten (b) sowie für Modell VI auch standardisierte Koeffizienten $(\beta)$. Signifikanz: $+p<0,10,{ }^{*} p<0,05$, $*^{*} p<0,01$, im Fall der Variablen im Block „Programmerfahrungen“ basierend auf einseitigen Tests

dung und Gemeinschaftserleben korrelieren nicht signifikant mit den Veränderungen im Selbstwert. Die individuellen Unterschiede in der Selbstwertschätzung am Ende des Programms lassen sich zu 46\% auf die Ausgangswerte zu Beginn der Exkursion und zu etwa $14 \%$ auf die während des Programms gemachten Erfahrungen zurückführen.

Die Veränderungen im emotionalen Wohlbefinden lassen sich ebenfalls systematisch auf zwei Erfahrungsbereiche während des Programms beziehen (Tab. 4): Zum einen hängt die Veränderung im Wohlbefinden wiederum sehr stark vom Kompetenzerleben ab: Wer sich bei den Aktivitäten als kompetent wahrnimmt, berichtet von positiveren Veränderungen im Wohlbefinden. Darüber hinaus ist die Gruppendynamik von Bedeutung, denn je stärker sich ein Jugendlicher bei den Aktivitäten mit den anderen als Gemeinschaft erlebte, desto positiver fällt die Veränderung im affektiven Wohlbefinden aus. Das Ausprobieren von neuen Aktivitäten oder von Aktivitäten, die eine Selbstüberwindung verlangen, ist indes kaum von Bedeutung, denn für beide Einflussfaktoren werden geringe, nicht-signifikante Effekte ausgewiesen. Insgesamt lassen sich die individuellen Unterschiede im subjektiven Wohlbefinden am Ende des Programms zu 13\% mit den Ausgangswerten erklären und zu 26\% mit den vier Variablen zum Programmerleben.

Über alle Modelle und Variablen hinweg zeigen sich in der Tendenz leicht negative Effekte für das Geschlecht. Auch wenn diese in den meisten Modellspezifikationen nicht signifikant ausfallen, legen sie insgesamt doch nahe, dass die Veränderungen im Verlauf des Programms bei weiblichen Jugendlichen im Durchschnitt etwas weniger positiv ausgeprägt sind als bei männlichen Jugendlichen. Auf ähnliche Effekte weist eine amerikanische Studie hin (Richmond et al. 2016), die zeigt, 
Tab. 4 Lineare Regressionsmodelle zur Vorhersage des affektiven Wohlbefindens am Ende des Programms ( $t 2)$ unter Kontrolle der Ausgangswerte $(t 1)$

\begin{tabular}{|c|c|c|c|c|c|c|c|}
\hline & \multicolumn{7}{|c|}{ Wohlbefinden ( $t 2)$} \\
\hline & I & II & III & IV & $\mathrm{V}$ & VI & VI $\beta$ \\
\hline \multicolumn{8}{|l|}{ Ausgangswerte } \\
\hline Wohlbefinden $(t 1)$ & $0,36^{* *}$ & $0,39 * *$ & $0,38 * *$ & $0,30 * *$ & $0,33 * *$ & $0,27 * *$ & 0,27 \\
\hline \multicolumn{8}{|l|}{ Programmerfahrungen } \\
\hline Neuartigkeit & - & 0,08 & - & - & - & 0,08 & 0,08 \\
\hline Selbstüberwindung & - & - & $-0,14$ & - & - & $-0,13$ & $-0,11$ \\
\hline Kompetenzerleben & - & - & - & $0,94 * *$ & - & $0,80 * *$ & 0,41 \\
\hline Gemeinschaftserleben & - & - & - & - & $0,59 * *$ & $0,32^{+}$ & 0,17 \\
\hline \multicolumn{8}{|l|}{ Kontrollvariablen } \\
\hline Alter & - & $-0,08$ & $-0,09$ & $-0,01$ & $-0,03$ & 0,01 & 0,01 \\
\hline Geschlecht & - & $-0,24$ & $-0,51$ & $-0,57$ & $-0,19$ & $-0,60$ & $-0,18$ \\
\hline Fam. Wohlstand & - & $-0,10$ & $-0,05$ & $-0,14$ & $-0,17$ & $-0,19$ & $-0,18$ \\
\hline $\mathrm{R}^{2}$ & 0,128 & 0,160 & 0,160 & 0,365 & 0,233 & 0,388 & \\
\hline $\begin{array}{l}\Delta \mathrm{R}^{2} \text { (im Vgl. zu } \\
\text { Modell I) }\end{array}$ & - & 0,032 & 0,032 & 0,237 & 0,105 & 0,260 & \\
\hline
\end{tabular}

Anmerkung: OLS Regression mit robusten Standardfehlern. Angegeben sind nicht-standardisierte Koeffizienten (b) sowie für Modell VI auch standardisierte Koeffizienten ( $\beta$ ). Signifikanz: $+p<0,10,{ }^{*} p<0,05$, $*^{*} p<0,01$, im Fall der Variablen im Block „Programmerfahrungen“ basierend auf einseitigen Tests

dass Jungen im Vergleich zu Mädchen in Outdoor-Gruppen eine höhere Wertschätzung zugesprochen wird, und zwar vor allem dann, wenn die Aktivitäten besonders risikoreich und physisch herausfordernd sind.

\section{Diskussion}

Der Beitrag hat basierend auf Prä- und Posttest-Daten von Jugendlichen und jungen Erwachsenen zum einen Veränderungen in den Selbstwirksamkeitserwartungen, im Selbstwert und im emotionalen Wohlbefinden aufgezeigt, die sich im Verlauf eines zehntägigen abenteuer- und erlebnispädagogischen Programms ergeben haben. Zum anderen wurden diese Veränderungen auf vier Einflussfaktoren zurückgeführt, um so Anhaltspunkte für Wirkungsweisen zu finden. Die Ergebnisse zeigen zunächst, dass die Teilnahme am Programm mit Veränderungen einherging, denn die Selbstwirksamkeitserwartungen $(d=0,33)$, der Selbstwert $(d=0,46)$ und das Wohlbefinden $(d=0,94)$ haben sich im Durchschnitt bei den Teilnehmenden erhöht. Die Effekte für Selbstwirksamkeit und Selbstwert liegen in etwa in der Größenordnung, die auch in Meta-Analysen berichtet wird (Hattie et al. 1997; Gillis und Speelman 2008). Der Effekt für das emotionale Wohlbefinden fällt etwas höher aus, allerdings unterliegt das Wohlbefinden auch stärkeren temporären Schwankungen, was z. B. an der vergleichsweise geringen Autokorrelation des Wohlbefindens über die Zeit abgelesen werden kann. Deshalb ist es nicht unwahrscheinlich, dass sich das Wohlbefinden der Teilnehmenden nach der Exkursion wieder in Richtung Ausgangswert zurückbewegt. 
Während ähnliche Effekte in früheren Studien berichtet wurden, gibt es kaum methodisch überzeugende Versuche, die Programmelemente und -erfahrungen zu identifizieren, die mit individuellen Veränderungen assoziiert sind. Hierzu haben wir einen Vorschlag gemacht und die Veränderungsvarianz zwischen Anreise $(t 1)$ und Abreise ( $t 2)$ auf vier Einschätzungen zurückgeführt: Neuigkeit, Selbstüberwindung, Kompetenzerleben und Bindung an die Gemeinschaft. Alle vier Aspekte gehören zu den Grundelementen erlebnispädagogischer Programme, die ja - idealtypisch betrachtet - Heranwachsende mit neuen, herausfordernden Aktivitäten konfrontieren sollen, die sie in der Gemeinschaft kompetent bewältigen können. Da diese Programmaspekte individuell verschieden erlebt werden, hatten wir vermutet, dass dieses Erleben mit individuellen Veränderungen zusammenhängt.

Die Befunde zeigen, dass sich einige, aber nicht alle der hierzu aufgestellten Hypothesen bestätigen lassen. Vor allem Kompetenzerlebnisse sind erwartungskonform und sehr eng mit positiven Veränderungen bei den Jugendlichen assoziiert (Hypothese 3). Darüber hinaus hängen positive Veränderungen in der Selbstwirksamkeit und im Selbstwert mit der Neuartigkeit der Aktivität zusammen (Hypothese 1); positive Veränderungen im affektiven Wohlbefinden hingegen mit der Qualität der Einbindung in die Gemeinschaft (Hypothese 4). Der oft in der Theoriedebatte fokussierte Aspekt der Selbstüberwindung ist indes weniger relevant und korreliert nicht mit Veränderungen bei den Teilnehmenden (Hypothese 2).

Diese Befunde zeigen, dass sich Wirkungen im Rahmen von erlebnispädagogischen Interventionen nicht pauschal ,erzeugen“ lassen, sondern im Zusammenhang mit individuellen Voraussetzungen und Prozessmerkmalen betrachtet werden müssen. Ein solche Prozessforschung ist aber ambitionierter als Ergebnisforschung (vgl. Heekerens 2018): Die gleiche Aktivität kann von Person 1 als bekannt und langweilig, Person 2 als neu und fordernd erlebt werden und Person 3 findet die Aktivität womöglich auch spannend, fühlt sich aber in der Gruppe nicht wohl. Die Ergebnisse dieses Beitrags legen aber nahe, dass diese Aspekte - die das „Erlebnis“, um das es ja geht, konstituieren - die Wirkung mitdefinieren. Aus pädagogischer Sicht besteht die zentrale Herausforderung darin, vor dem Hintergrund heterogener Ausgangslagen einzelne Programmbausteine und -aktivitäten so vielfältig und differenziert zu gestalten, dass möglichst alle Teilnehmenden eine für sie neue, aber auch zu bewältigende Aufgabe finden, bei der sie sich als kompetent erleben. Im Gegensatz dazu ist zumindest vorläufig anzunehmen, dass der in theoretischen Modellen zur Erlebnispädagogik beschriebene intrapersonale Spannungszustand, der sich in anfänglicher Unsicherheit und Selbstüberwindung äußert, nicht konstitutiv für einen Programmeffekt ist.

Zwei Anmerkungen sind unserer Ansicht nach in diesem Zusammenhang bedeutsam: Zum einen liegt es auf der Hand, dass Kompetenzerlebnisse wirkungslos bleiben, wenn die Aufgabe, bei der sich eine Person kompetent fühlt, trivial ist. Bei den hier untersuchten Aktivitäten kann das nahezu ausgeschlossen werden, weil diese - egal ob Kanuwanderung, Höhlenexpedition oder Kletteraktivität - für Jugendliche fast immer einen gewissen Abenteuercharakter aufweisen, und zwar auch dann, wenn man nicht (anfangs) an seinen Fähigkeiten zur Bewältigung dieser Aktivitäten zweifelt. Zum anderen lässt sich die Frage aufwerfen, ob der in den theoretischen Modellen zur Erlebnispädagogik beschriebene intrapersonale Spannungs- 
zustand (vgl. Deane und Harré 2013) tatsächlich als unabhängiger Einflussfaktor auf die Programmwirksamkeit zu verstehen ist. Es ließe sich auch annehmen, dass der Effekt des Kompetenzerlebens auf Persönlichkeitsveränderungen vom Grad der Selbstüberwindung moderiert wird: Je mehr sich eine Person vor einer Aktivität überwinden muss, desto stärker wird das Kompetenzerleben bei der Aktivität eine Veränderung im Selbstbild bewirken. In weiterführenden (hier nicht dokumentierten) Regressions- und Varianzanalysen haben wir diesen Moderationseffekt geprüft. Diese Modelle weisen zwar keinen signifikanten Effekt aus, die Tendenzen zeigen aber in die erwartete Richtung: So ist der Effekt des Kompetenzerlebens auf die Veränderung im Selbstwert z. B. stärker bei den Jugendlichen, die ein hohes Maß an Selbstüberwindung angegeben haben. Für die dezidierte Prüfung komplexerer Modellannahmen sind aber größere Stichproben vonnöten.

Zum Schluss sind einige Limitationen dieser Studie zu erwähnen, die aber typisch für das Forschungsfeld an sich sind: Erstens basiert unsere Studie auf einer kleinen Stichprobe. Auch wenn wir Daten mehrerer Jugendgruppen, die das gleiche zehntägige Programm durchlaufen haben, kombinieren konnten, bleibt die Datenbasis überschaubar. Erlebnispädagogische Programme finden immer in Kleingruppen statt, weshalb quantitative Analysen selten auf größere Fallzahlen zurückgreifen können (vgl. hierzu auch den Forschungsüberblick in Deane und Harré 2013). Andererseits impliziert dies, dass Effekte tatsächlich erst ab einer substantiellen Größenordnung als signifikant ausgewiesen werden und somit immer von praktischer und pädagogischer Relevanz sind. Zweitens hat der Beitrag vier Bedingungen fokussiert, was aber nicht bedeutet, dass es keine weiteren Einflüsse gibt, die mit individuellen Veränderungen während eines Programms zusammenhängen. Zudem gibt es bei erlebnispädagogischen Aktivitäten eine Vielzahl spezifischer Einflüsse (z. B. einzelne Entscheidungen der Gruppe, die den weiteren Verlauf der Aktivität prägen), die ebenfalls bedeutsam sind, sich aber standardisiert nicht erheben lassen. Im Fall des hier untersuchten Programms konnte zumindest sichergestellt werden, dass Ablauf, Aktivitäten und Anleitung durch das Personal vor Ort in allen Gruppen auf sehr ähnliche Art und Weise stattgefunden hat. Eine weitere Standardisierung ist kaum möglich ohne die ökologische Validität solcher Studien zu beeinträchtigen. Drittens haben wir keinen Messzeitpunkt nach der Ferienfreizeit als Follow-up realisieren können, weil die Teilnehmenden nur vor Ort im Camp befragt werden konnten. Entsprechend sind die Aussagen der Studie auf kurzfristige Veränderungen beschränkt. Wie lang die Veränderungen anhalten, lässt sich auf Basis der Studie nicht beantworten. Reviews zeigen aber, dass Effekte, die zumindest über mehrere Wochen nachweisbar sind, keine Seltenheit sind und diese manchmal sogar stärker ausfallen als direkt nach Ende des Outdoor-Programms (Hattie et al. 1997). Gleichwohl wäre es wünschenswert, wenn zukünftige Arbeiten die Frage nach Langzeiteffekten aufgreifen würden. Wünschenswert wären zudem weitere Messzeitpunkte während des Programms, um Veränderungsverläufe detaillierter beschreiben zu können.

Schließlich ist auf Einschränkungen hinzuweisen hinsichtlich der Deutung der Veränderungen von $t 1 \mathrm{zu} t 2$ als Wirksamkeit des Programms. Das Fehlen einer randomisierten Kontrollgruppe schränkt die Möglichkeiten ein, eine Wirksamkeit nachzuweisen. So bleibt unklar, inwieweit die aufgezeigten Veränderungen spezifisch für das hier untersuchte abenteuerpädagogische Programm sind oder sich ähn- 
liche Muster auch bei anderen Urlaubsreisen und Ferienfreizeiten finden lassen. Die Frage nach der Wirksamkeit stand deshalb auch nicht im Fokus des Beitrags. Für die dargestellten Modelle zum Zusammenhang von Erfahrungen, die im Programm gemacht wurden, und den Veränderungen, die sich im Programm ergeben haben, ist das Fehlen einer Kontrollgruppe weniger entscheidend. Allerdings basieren die Modelle auf einem korrelativen Ansatz, der zwar die zeitliche Ordnung von vermeintlicher Ursache und vermeintlicher Wirkung beachtet, jedoch nicht für alle denkbaren Drittvariablen kontrolliert (vgl. hierzu auch Reinders 2006). Es ließen sich möglicherweise noch weitere Prozessmerkmale identifizieren und erheben, die ebenfalls relevant sind. Abhängig von der Stärke der Interkorrelation der Prozessmerkmale könnte dies zu einer Veränderung der Modellparameter führen. Gleichwohl geht unser Design über die bislang vorliegenden nationalen und internationalen Arbeiten zur Prozess-Ergebnis-Forschung in der Abenteuerpädagogik deutlich hinaus, weil Veränderungen nicht retrospektiv von den Teilnehmenden bewertet werden (wie z. B. bei Paisley et al. 2008; Sibthorp et al. 2007, 2011), sondern auf den gemessenen Veränderungen vom Anreise- zum Abreisetag basieren.

Funding Open Access funding provided by Projekt DEAL.

Open Access Dieser Artikel wird unter der Creative Commons Namensnennung 4.0 International Lizenz veröffentlicht, welche die Nutzung, Vervielfältigung, Bearbeitung, Verbreitung und Wiedergabe in jeglichem Medium und Format erlaubt, sofern Sie den/die ursprünglichen Autor(en) und die Quelle ordnungsgemäß nennen, einen Link zur Creative Commons Lizenz beifügen und angeben, ob Änderungen vorgenommen wurden.

Die in diesem Artikel enthaltenen Bilder und sonstiges Drittmaterial unterliegen ebenfalls der genannten Creative Commons Lizenz, sofern sich aus der Abbildungslegende nichts anderes ergibt. Sofern das betreffende Material nicht unter der genannten Creative Commons Lizenz steht und die betreffende Handlung nicht nach gesetzlichen Vorschriften erlaubt ist, ist für die oben aufgeführten Weiterverwendungen des Materials die Einwilligung des jeweiligen Rechteinhabers einzuholen.

Weitere Details zur Lizenz entnehmen Sie bitte der Lizenzinformation auf http://creativecommons.org/ licenses/by/4.0/deed.de.

\section{Literatur}

Amesberger, G. (1998). Persönlichkeitsentwicklung durch Outdoor-Aktivitäten? Untersuchung zur Persönlichkeitsentwicklung und Realitätsbewältigung bei sozial Benachteiligten. Frankfurt: Afra.

Arahanga-Doyle, H., Moradi, S., Brown, K., Neha, T., Hunter, J. A., \& Scarf, D. (2019). Positive youth development in Māori and New Zealand European adolescents through an adventure education programme. Kōtuitui: New Zealand Journal of Social Sciences Online, 14(1), 38-51.

Boeger, A., Dorfler, T., \& Schut-Ansteeg, T. (2006). Project adventure with adolescents: influence on psychopathology and self-esteem. Praxis der Kinderpsychologie und Kinderpsychiatrie, 55(3), 181-197.

Bradburn, N. M. (2015). The affect balance scale. In W. Glatzer, L. Camfield, V. Møller \& M. Rojas (Hrsg.), Global handbook of quality of life (S. 269-279). Heidelberg: Springer.

Brand, D. (2002). A longitudinal study of the effects of a wilderness-enhanced program on behaviourdisordered adolescents. Journal of Outdoor and Environmental Education, 6(1), 40-56.

Brown, M. (2008). Comfort Zone: model or metaphor? Australian Journal of Outdoor Education, 12(1), $3-12$.

Cook, E. C. (2008). Residential wilderness programs: The role of social support in influencing self-evaluations of male adolescents. Adolescence, 43(172), 751-774. 
Cordle, J., Van Puymbroeck, M., Hawkins, B., \& Baldwin, E. (2016). The effects of utilizing high element ropes courses as a treatment intervention on self-efficacy. Therapeutic Recreation Journal, 50(1), 75-92.

D’Amato, L. G., \& Krasny, M.E. (2011). Outdoor adventure education: applying transformative learning theory to understanding instrumental learning and personal growth in environmental education. Journal of Environmental Education, 42(4), 237-254.

Deane, K. L., \& Harré, N. (2013). The youth adventure programming model. Journal of Research on Adolescence, 24(2), 293-308.

Draper, C. E., Lund, C., \& Flisher, A. J. (2011). A retrospective evaluation of a wilderness-based leadership development program. South African Journal of Psychology, 41(4), 451-464.

Duerden, M.D., \& Witt, P. A. (2010). The impact of socialization on youth program outcomes: A social development model perspective. Leisure Sciences, 32(4), 299-317.

Ewert, A.W., \& McAvoy, L. (2000). The effects of wilderness settings on organized groups: a state-ofknowledge paper. In S. McCool, D. Cole, W. Borrie \& J. O’Loughlin (Hrsg.), Wilderness science in a time of change. Conference Proceedings. (S. 13-26). Ogden: USDA.

Fengler, J. (2017). Zur Frage des Forschungsstandes in der Erlebnispädagogik. Zeitschrift für Bildung und Erziehung. Themenheft „Erlebnispädagogik in Theorie und Praxis“, 3, 319-334.

Ferring, D., \& Filipp, S.-H. (1996). Messung des Selbstwertgefühls: Befunde zu Reliabilität, Validität und Stabilität der Rosenberg-Skala. Diagnostica, 42(3), 284-292.

Fischer, T., \& Ziegenspeck, J.W. (2008). Erlebnispädagogik: Grundlagen des Erfahrungslernens. Bad Heilbrunn: Klinkhardt.

Gatzemann, T., Schweizer, K., \& Hummel, A. (2008). Effectiveness of sports activities with an orientation on experiential education, adventure-based learning and outdoor-education. Kinesiology, 40(2), 146-152.

Gehris, J., Kress, J., \& Swalm, R. (2010). Students' views on physical development and physical selfconcept in adventure-physical education. Journal of Teaching in Physical Education, 29(2), 146-166.

Gillespie, E., \& Allen-Craig, S. (2009). The enhancement of resilience via a wilderness therapy program: a preliminary investigation. Australian Journal of Outdoor Education, 13(1), 39-49.

Gillis, H.L., \& Gass, M. A. (2010). Treating juveniles in a sex offender program using adventure-based programming: a matched group design. Journal of Child Sexual Abuse, 19(1), 20-34.

Gillis, L.H., \& Speelman, E. (2008). Are challenge (ropes) courses an effective tool? A meta-analysis. Journal of Experiential Education, 31(2), 111-135.

Goldenberg, M., McAvoy, L., \& Klenosky, D.B. (2005). Outcomes from the components of an Outward Bound experience. Journal of Experiential Education, 28(2), 123-146.

Hattie, J., Marsh, H.W., Neill, J. T., \& Richards, G. E. (1997). Adventure education and outward bound: out-of-class experiences that make a lasting difference. Review of Educational Research, 67(1), 43-87.

Hayhurst, J., Hunter, J. A., Kafka, S., \& Boyes, M. (2015). Enhancing resilience in youth through a 10-day developmental voyage. Journal of Adventure Education and Outdoor Learning, 15(1), 40-52.

Heckmair, B., \& Michl, W. (2008). Erleben und Lernen. Einführung in die Erlebnispädagogik. München: Ernst Reinhardt Verlag.

Heekerens, H.-P. (2006). Wirksamkeitsforschung zur Erlebnispädagogik: Ergebnisse, Fragen, Anregungen. Zeitschrift für Erlebnispädagogik, 26(10), 3-57.

Heekerens, H.-P. (2018). Ergebnis- und Prozessforschung in der Erlebnispädagogik. In W. Michl \& H. Seidel (Hrsg.), Handbuch Erlebnispädagogik (S. 314-321). München: Reinhardt.

Hinds, J. (2011). Exploring the psychological rewards of a wilderness experience. An interpretive phenomenological analysis. The Humanistic Psychologist, 39(3), 189-205.

Hopkins, D., \& Putnam, R. (1997). Personal growth through adventure. London: David Fulton.

Hunter, J. A., Hayhurst, J., Kafka, S., Boyes, M., Ruffman, T., O’Brien, K., \& Stringer, M. (2013). Elevated self-esteem 12 months following a 10-day developmental voyage. Journal of Applied Social Psychology, 43(9), 1956-1961.

Kimball, R. O., \& Bacon, S. B. (1993). The wilderness challenge model. In M. A. Gass (Hrsg.), Adventure therapy (S. 11-41). Dubuque: Kendall/Hunt.

Kinne, T., \& Theunissen, G. (Hrsg.). (2013). Erlebnispädagogik in der Behindertenarbeit. Stuttgart: Kohlhammer.

Klawe, W. (2006). Wie wirken pädagogische Interventionen? Zur Wirkungsforschung bei individualpädagogischen Maßnahmen. Erleben \& lernen, 2006(3-4), 6-11.

Kolb, D. (1984). Experiential learning: experience as the source of learning and development. Englewood Cliffs: Prentice Hall. 
Kümmel, U., Hampel, P., \& Meier, M. (2008). Einfluss einer erlebnispädagogischen Maßnahme auf die Selbstwirksamkeit, die Stressverarbeitung und den Erholungs-Beanspruchungs-Zustand bei Jugendlichen. Zeitschrift für Pädagogik, 54(4), 555-571.

Lakemann, U. (2017). Die Wurzeln erfahrungsorientierter Therapie: Stand der internationalen Forschung. In K. Mehl (Hrsg.), Erfahrungsorientierte Therapie: Integrative Psychotherapie und moderne Psychosomatik (S. 71-109). Wiesbaden: Springer VS.

Luckner, J.L., \& Nadler, R.S. (1997). Processing the experience: strategies to enhance and generalize learning. Dubuque: Kendall/Hunt Publishing.

Martin, A. J., \& Leberman, S. I. (2005). Personal learning or prescribed educational outcomes: a case study of the outward bound experience. Journal of Experiential Education, 28(1), 44-59.

McDonald, M., Wearing, S., \& Ponting, J. (2009). The nature of peak experience in wilderness. The Humanistic Psychologist, 37(4), 370-385.

McKenzie, M.D. (2000). How are adventure education program outcomes achieved? A review of the literature. Australian Journal of Outdoor Education, 5(1), 19-27.

McKenzie, M.D. (2003). Beyond ,the outward bound process": rethinking student learning. Journal of Experiential Education, 26(1), 8-23.

Moch, M. (2002). Entwicklung von Gruppenstruktur, Zusammenhalt und Selbstvertrauen im Verlauf erlebnispädagogischer Segelmaßnahmen. Gruppendynamik und Organisationsberatung, 33(1), 83-95.

Mutz, M., \& Müller, J. (2016). Mental health benefits of outdoor adventures: results from two pilot studies. Journal of Adolescence, 49, 105-114.

Mutz, M., Müller, J., \& Göring, A. (2019). Effects of outdoor adventures on adolescents' mental health: daily screen time as a moderator of changes. Journal of Adventure Education \& Outdoor Learning, $19(1), 56-66$.

Nadler, R. S. (1993). Therapeutic process of change. In M. A. Gass (Hrsg.), Adventure therapy (S. 57-69). Dubuque: Kendall/Hunt.

Newsom, J.T. (2012). Basic longitudinal analysis approaches for continuous and categorical variables. In J. T. Newsom, R. N. Jones \& S. M. Hofer (Hrsg.), Longitudinal data analysis (S. 143-180). New York: Routledge.

Nichols, G. (2000). Risk and adventure education. Journal of Risk Research, 3(2), 121-134.

Öhman, J., \& Sandell, K. (2016). Environmental concerns and outdoor studies: nature as fosterer. In B. Humberstone, H. Prince \& K. A. Henderson (Hrsg.), Routledge International Handbook of Outdoor Studies (S. 30-39). New York: Routledge.

Paisley, K., Furman, N., Sibthorp, J., \& Gookin, J. (2008). Student learning in outdoor education: a case study from the national outdoor leadership school. Journal of Experiential Education, 30(3), 201-222.

Panicucci, J. (2007). Cornerstones of adventure education. In D. Prouty, J. Panicucci \& R. Collinson (Hrsg.), Adventure education (S. 33-48). Champaign: Human Kinetics.

Priest, S. (1999). Research in Outdoor Adventure. In F. H. Paffrath, A. Salzmann \& M. Scholz (Hrsg.), Wissenschaftliche Forschung in der Erlebnispädagogik. Erleben, Forschen, Evaluieren (S. 13-23). Augsburg: ZIEL.

Reichmann, M. (2011). Förderung von Sozialkapital, Gruppenkohäsion und Psychological Sense of Community in Unternehmen durch erlebnispädagogische Trainingsprogramme. Konstanz: Universitätsverlag.

Reinders, H. (2006). Kausalanalysen in der Längsschnittforschung. Das Cross-Lagged-Panel-Design. Diskurs Kindheits- und Jugendforschung, 1(4), 569-587.

Reuker, S. (2009). Sozialerzieherische Wirkungen schulischer Bewegungsangebote. Eine empirische Studie zu erlebnispädagogischen Schulfahrten. Sportwissenschaft, 39(4), 330-338.

Richmond, D., Sibthorp, J., Jostad, J., \& Gookin, J. (2016). Factors determining peer status in outdoor adventure groups. Journal of Outdoor Recreation, Education \& Leadership, 8(1), 41-56.

Rosenberg, M. (1965). Society and the adolescent self-image. Princeton: Princeton University Press.

Scarf, D., Hayhurst, J. G., Riordan, B.C., Boyes, M., Ruffman, T., \& Hunter, J. A. (2017). Increasing resilience in adolescents: the importance of social connectedness in adventure education programmes. Australasian Psychiatry, 25(2), 154-156.

Scarf, D., Kafka, S., Hayhurst, J., Jang, K., Boyes, M., Thomson, R., \& Hunter, J. A. (2018). Satisfying psychological needs on the high seas: explaining increases self-esteem following an Adventure Education Programme. Journal of Adventure Education and Outdoor Learning, 18(2), 165-175.

Schell, L., Cotton, S., \& Luxmoore, M. (2012). Outdoor adventure for young people with mental illness. Early Intervention in Psychiatry, 6(4), 407-414. 
Schnell, R., Bachteler, T., \& Reiher, J. (2010). Improving the use of self-generated identification codes. Evaluation Review, 34(5), 391-418.

Schwarzer, R., \& Jerusalem, M. (Hrsg.). (1999). Skalen zur Erfassung von Lehrer- und Schülermerkmalen. Berlin: Freie Universität Berlin.

Sibthorp, J. (2003). An empirical look at Walsh and Golins' adventure education process model: relationships between antecedent factors, perceptions of characteristics of an adventure education experience, and changes in self-efficacy. Journal of Leisure Research, 35(2), 80-106.

Sibthorp, J., Furmann, N., Paisley, K., Gookin, J., \& Schumann, S. (2011). Mechanisms of learning transfer in adventure education: qualitative results from the NOLS transfer survey. Journal of Experiential Education, 34(2), 109-126.

Sibthorp, J., Paisley, K., \& Gookin, J. (2007). Exploring Participant development through adventure-based recreation programming: a model from the National Outdoor Leadership School. Leisure Sciences, 29(1), 1-18.

Sommer, S. (2005). Erlebnispädagogik in der offenen Jugendarbeit. Eine Untersuchung möglicher Wirkungen. In U. Lakemann (Hrsg.), Wirkungsimpulse von Erlebnispädagogik und Outdoor- Training (S. 108-128). Augsburg: Ziel.

Stott, T., Allison, P., Felter, J., \& Beames, S. (2015). Personal development on youth expeditions: a literature review and thematic analysis. Leisure Studies, 34(2), 197-229.

Stremba, B., \& Bisson, C. (Hrsg.). (2009). Teaching adventure education theory - Best practises. Champaign: Human Kinetics.

Tambs, K., \& Røysamb, E. (2014). Selection of questions to short-form versions of original psychometric instruments in MoBa. Norsk Epidemiologi, 24(1-2), 195-201.

Walsh, V., \& Golins, G. (1976). The exploration of the Outward Bound process. Denver: Colorado Outward Bound School. 